\title{
The extracellular glycosphingolipid-binding motif of Fas defines its internalization route, mode and outcome of signals upon activation by ligand
}

\author{
K Chakrabandhu ${ }^{*, 1}$, S Huault ${ }^{1}$, N Garmy ${ }^{2}$, J Fantini ${ }^{2}$, E Stebe ${ }^{1}$, S Mailfert ${ }^{3,4,5}$, D Marguet ${ }^{3,4,5}$ and A-O Hueber ${ }^{\star, 1}$
}

Selective compartmentalization and internalization have been shown as a means for regulating specific signals of cell surface receptors to correspond to cellular requirements and conditions. Here, we present a conserved extracellular glycosphingolipidbinding motif of Fas as one of the regulatory elements in the selection of its internalization route and consequently the signals transmitted upon ligand binding. This motif is required for clathrin-mediated internalization of Fas, which allows the transduction of its cell death signal. The loss of function of the motif drives the activated receptor to an alternative internalization route that is independent of clathrin and cholesterol-dependent rafts but dependent on ezrin, and thereby extinguishing its cell death signal while promoting its non-death functions. Through biochemical, biophysical, and genetic approaches, we present a protein/ lipid-based mechanism as a key to the versatility of the signal transduction by the multifunctional Fas receptor-ligand system. Cell Death and Differentiation (2008) 15, 1824-1837; doi:10.1038/cdd.2008.115; published online 1 August 2008

Fas (CD95/APO-1/TNFRSF6), a TNF receptor superfamily member, is well known as a key apoptosis activator. The binding with its ligand (FasL) leads to the death-inducing signalling complex (DISC) formation upon the recruitment of FADD and procaspase-8, the caspase cascade activation, and cell death. ${ }^{1}$ In addition, Fas can also induce non-apoptotic activities, for example, tissue regeneration and proliferation. ${ }^{2}$ However, little has been described about the regulation of its adaptable functions.

Studies have indicated endocytosis as a means for coordinating the type, extent, and timing of signals of surface receptors. ${ }^{3,4}$ This is the case for Fas, of which efficient cell death signal requires its internalization ${ }^{5,6}$ and this process involves the interaction between Fas and its lipidic environment including its targeting by palmitoylation to the cholesterol-, glycosphingolipid-rich membrane microdomains (rafts). ${ }^{6}$

A glycosphingolipid has an oligosaccharide headgroup linked to a ceramide backbone. Many glycosphingolipids are involved in the sorting and trafficking of proteins and lipids. ${ }^{7,8}$ Recently, a common motif with glycosphingolipid-binding specificity has been identified in proteins that share little sequence homology. ${ }^{9}$ Here, we report the identification of such a motif in Fas and its role in the selection of the internalization route of the receptor, which defines its mode of signal.
Results

The extracellular domain of Fas contains a glycosphingolipid-binding motif. An extracellular motif in CRD2 and CRD3 regions of Fas shares some sequence similarity to the V3 loop of HIV-1 gp120, which has been reported as (glyco)sphingolipid-binding domain $(\mathrm{SBD})^{9}$ (Figure 1a). Our comparative modelling further shows a striking structural similarity between this motif and the V3 loop (Figure 1b).

This putative glycosphingolipid-binding motif (GBM) is conserved in Fas across species and among TNFR superfamily members (Figure 1c and d, Supplementary Figure S1). As the primary sequence indicates its proximity to the ligandinteracting surface, ${ }^{10,11}$ we examined its location with respect to the FasL-binding site in a structural context. As the crystal structure of Fas-FasL complex has not been resolved, we constructed a structural model of Fas-FasL complex based on the three-dimensional structures of TNFR1, TNF ${ }_{\alpha}$, and TNF ${ }_{\beta}$, as described for the identification of FasL-binding site. ${ }^{11}$ The GBM forms a loop downstream from the FasL-binding surface, with two aromatic residues (F133 and F134) at the turn (Figure 1e). This structure coincides with that of the V3 SBD which forms a hairpin structure with aromatic residues at the turn, and thus allowing the protein-

\footnotetext{
${ }^{1}$ Equipe labelisée La Ligue; Institute of Developmental Biology and Cancer Research, CNRS UMR 6543, Nice, France; ${ }^{2}$ Laboratoire des Interactions Moléculaires et Systèmes Membranaires, CNRS UMR 6231, Faculté des Sciences et Techniques St-Jérôme, Université Paul Cézanne, Marseille, France; ${ }^{3}$ Centre d'Immunologie de Marseille Luminy, Université de la Méditerranée, Marseille, France; ${ }^{4}$ INSERM, UMR 631, Marseille, France and ${ }^{5} \mathrm{CNRS}$, UMR 6102, Marseille, France ${ }^{*}$ Corresponding authors: K Chakrabandhu or A-O Hueber, Institute of Signaling, Developmental Biology and Cancer Research, CNRS UMR 6543, Centre A. Lacassagne, 33, Avenue de Valombrose, 06189 Nice, France. Tel: + 33 492031241; Fax: + 33 492031245; E-mail: hueber@unice.fr or chakraba@unice.fr Keywords: cell death; Fas; glycosphingolipid; signal transduction

Abbreviations: BCR, B-cell receptor; CALM, clathrin assembly lymphoid myeloid leukaemia protein; CHC, clathrin heavy chain; CME, clathrin-mediated endocytosis; CRD, cysteine-rich domain; EGFR, epidermal growth factor receptor; FADD, Fas-associated death domain protein; FasL, Fas ligand; GBM, glycosphingolipid-binding motif; Gb3, globotriosylceramide; Gb4, globoside; GD3, disialoganglioside; LacCer, lactosylceramide; LDLR, low density lipoprotein receptor; rED-Fas, recombinant extracellular domain of Fas; shRNAi, short hairpin RNA interference; TNF, tumour-necrosis factor; TNFR, tumour-necrosis factor receptor; TfR, transferrin receptor; $\pi_{\mathrm{i}}$, initial surface pressure; $\Delta \pi$, surface pressure increase

Received 12.11.07; revised 11.6.08; accepted 18.6.08; Edited by A Ashkenazi; published online 01.8 .08
} 
glycosphingolipid interaction through the stacking of the aromatic ring of the amino acid against the sugar ring of glycosphingolipid. ${ }^{12,13}$

Fas GBM requires its aromatic residues to bind to selected glycosphingolipids. The interaction between the extracellular domain of Fas and glycosphingolipids was demonstrated by analysing the interaction between the recombinant extracellular domain of Fas (rED-Fas) and lipid monolayers using the Langmuir film balance technique. The rED-Fas interacted strongly with the glycosphingolipids LacCer and Gb3 and weakly with GD3 (Figure 2a). As GBM may be responsible for this interaction, we analysed the interaction between a peptide derived from the GBM of murine Fas (GBM-mFas) and lipid monolayers. Similarly, the peptide interacted strongly with LacCer and Gb3 but very weakly with GD3 and Gb4 (Figure 2b) and did not interact with the sphingomyelin, which lacks a sugar head group (not shown). These results indicate that the interactions between the extracellular domain of Fas and certain glycosphingolipids could be mediated by the specific glycosphingolipid recognition through GBM.

GBM-mFas/LacCer docking simulation (Figure 2c) showed Y130 stacking against the galactose ring of LacCer, mediating the $\mathrm{CH}-\pi$ interaction that is driven by the proximity of the aliphatic protons of the sugar ring and the $\pi$-electron cloud of the aromatic ring. ${ }^{13,14}$ This was also observed for GBM of human Fas (hFas, Supplementary Figure S1). Because the lipid monolayer becomes less compressible as the initial pressure $\left(\pi_{\mathrm{i}}\right)$ increases, if the peptide binds specifically to the monolayer, the $\Delta \pi$ will decrease as the $\pi_{\mathrm{i}}$ of the monolayer increases $^{15}$ (see Supplementary Information). Figure 2d demonstrates a specific interaction between the GBM-mFas and LacCer, which was disrupted when aromatic residues in the GBM were alanine-substituted (shown by the $\Delta \pi$ drop). Similar results were observed with the corresponding hFas peptides (Supplementary Figure S1). In addition, Fas GBM also bound to suramin (Figure 2e), a polysulfonyl naphtylurea that inhibits the binding of HIV-1 gp120 to GalCer, its receptor in human colon epithelial cells. ${ }^{16}$ Although both aromatic residues of the GBM contributed to the binding, the $Y 130$ of $\mathrm{mFas}$ (or $\mathrm{F} 134$ of hFas) was the greater contributor given a stronger binding inhibition upon the $\mathrm{Y} \rightarrow \mathrm{A}$ substitution (mFas, Figure 2e; hFas, Supplementary Figure S1). These results present a potential functional significance of the GBM and indicate that its mechanism of protein-lipid interaction may resemble that of the V3 loop.

The GBM is essential for Fas-induced cell death. Following cell-free studies, we further investigated GBM's functions at the cellular level. Stable cell lines expressing mFas or mFas with GBM mutations (F129A, $\mathrm{Y} 130 \mathrm{~A}$, or both) at equivalent level were subjected to FasLtriggered cell death assay. Although the F129A mutation resulted in a significant cell death reduction, a stronger death inhibition was due to the mutations Y130A and F129A/Y130A (Figure $3 a$ and $b$ ). This was confirmed by the reduction of the cleavage of caspase 9, Bid, and PARP, with the mFas. Y130A and mFas.F129A/Y130A exerting a greater reduction (Figure $3 \mathrm{c}$ ). The loss of aromatic residues in the
GBM also resulted in cell death inhibition when the cells were cocultured with FasL-expressing cells (Figure 3d). The reduced sensitivity to Fas-mediated apoptosis in GBMdefective cells was not due to a receptor-ligand interaction defect as these cells had equivalent FasL-binding ability (Supplementary Figures S2 and S3). Altogether, these results indicate that the GBM is essential for Fas cell death signalling and that the $\mathrm{Y} 130$ is the most critical aromatic residue for its function. Accordingly, studies presented henceforth were conducted with the mFas.Y130A or hFas.F134A representing GBM-defective Fas.

GBM plays a central role in Fas non-death signalling. Because Fas also signals non-death pathways, ${ }^{2}$ we investigated GBM's role in some Fas-induced non-death responses. Upon sublethal stimulation with FasL, the cells exhibited a decreased motility, which was more pronounced in L1210.mFas. Y130A cells (Figure 4a). The stimulation with FasL also caused an increased p42/p44 MAPK activation (Figure 4b), and proliferation (Figure $4 \mathrm{c}$ and d) in L1210.mFas. Y130A cells when compared with the L1210.mFas cells. Similarly, this was observed for cells expressing corresponding hFas constructs (Supplementary Figure S3).

These results indicate that the GBM is indispensable for the cell death signal of the ligand-activated Fas and that its absence allows certain non-death signal surges. This therefore presents the role of Fas-glycosphingolipid interaction as a mechanism controlling the signalling modes of the Fas/FasL system.

The GBM participates in the membrane dynamics of Fas. To identify the level at which the GBM exerts its control on Fas signalling, we examined the initial events of FasLtriggered Fas-mediated signalling. We previously reported that the targeting of Fas to rafts by palmitoylation is essential for its efficient cell death signal. ${ }^{2,6}$ As glycosphingolipids are major constituents of rafts, we investigated the role of the GBM in Fas localization to the rafts. Immunoblots of fractions from the detergent-resistant membrane microdomain (DRM) preparations (boiled in Laemli buffer prior to SDS-PAGE) showed that the DRM-residing Fas in L1210.mFas cells was mostly detected as lower molecular weight (MW) bands in fractions 3 and 4 , with barely detectable bands of the SDS-, $\beta$-mercaptoethanol-resistant, higher-MW (hereafter called SDS-stable) oligomers $(\sim 100 \mathrm{kDa})$ localized in the lowest density fractions of the gradient (fractions 1 and 2) (Figure 5a). In contrast, the majority of the DRM-residing Fas in L1210.mFas.Y130A cells was detected as SDS-stable oligomers in fractions 1 and 2, and the proportion of Fas of lower MW bands in fractions 3 and 4 was clearly reduced. The role of the GBM in the SDS-stable oligomer formation was supported by the data from breast cancer epithelial cells (CAL51) where a clear increase in higher order oligomers of Fas was observed upon overexpressing hFas.F134A but not hFas (Figure 5b).

Considering the following observations: (1) the mFas. Y130A localized in the lowest density fractions of the DRM preparation with the majority of DRM-residing portion being detected as SDS-stable oligomers and (2) the increased in the higher order oligomers of hFas.F134A in CAL51 cells; 
it is possible that, rather than in raft targeting, the GBM's importance may be in the dynamic multi-molecular organization of Fas.
Subsequently, we investigated the lateral diffusion of hFas.GFP and the GBM-defective hFas.F134A.GFP molecules on the plasma membrane of live COS-7 cells by

a

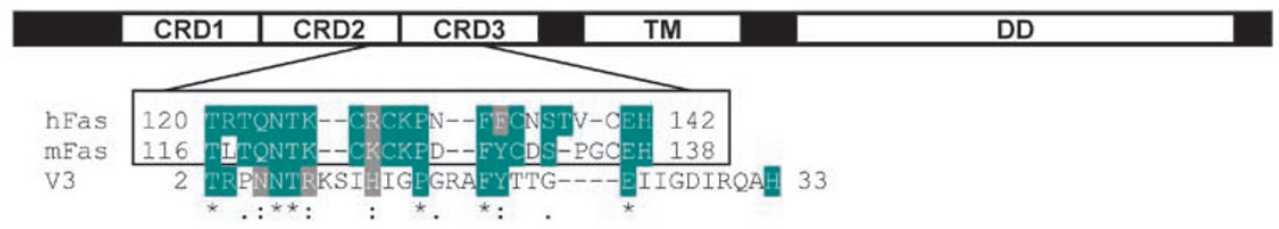

b
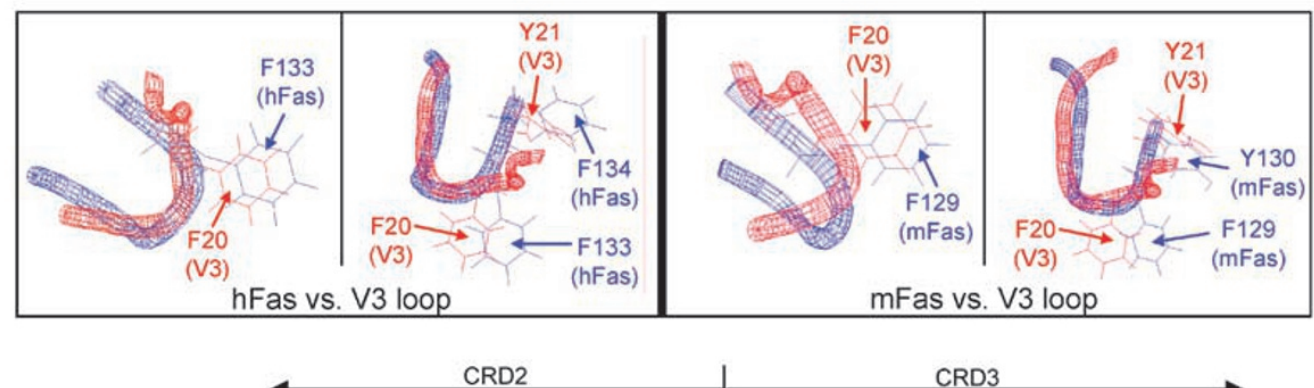

mFas vs. V3 loop

c Human

Chimpanze

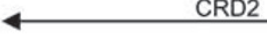

CRD3

115 KCRRCRLCDEGHGLEVEINCTRTQNTKCRCKPNE

sus monkey 100 KCRRCRLCDEGHGLEVEINCTRTQNTKCRCKPNFFC--NSAV----CEHCDPCTKC-KHG 152

Pig

Bovine

Cat

Dog

Norway rat

Mouse

Chicken

Medaka fish

Zebrafish

100 KCRRCRLCDEGHGLEVEINCTRTQNTKCRCKPNEFC--NSTV----CEHCDPCTKC-EHG 152

-NSTV----CEHCDPCTKC-EHG 167

98 KCRRCRVCDGEHGLEVEKNCTRTQNTKCRCKPNEFC--HTSQ----CEHCNPCTTC-EHG 150

97 KCIRCSICDEEHGLEVEQNCTRTRNTKCRCKSNEEC--NSSP----CEHCNPCTTC-EHG 149

100 RCRRCKICDEEHGLEVEKNCTRTONTKCRCKSNFEC--NVSQ----CDHCNPCMMC-EHG 152

82 KCRRCGICDGEHGLEVERNCTQRRNTKCRCKPNFYC--DVSP----CEHCNPCSTC-EHG 135

96 KCRRCAFCDEGHGLEVETNCTRTQNTKCRCKENEYC--NASL----CDHCYHCTSCGLED 149

96 KCRRCTLCDEEHGLEVETNCTITQNTKCKCKEDEYC--DSPG----CEHCVRCASC-EHG 148

45 ECMRCRSCDKALGLEVVKNCTSIENAE S AAKNHYC--NSSR----CEHCESCTVCENGQ 98

102 QCLLCKRCDPEQGEDVHSPCIVERNTV CKCKVNEFCGTNSIQDPRS CDHCQPCTQC-EKG 160

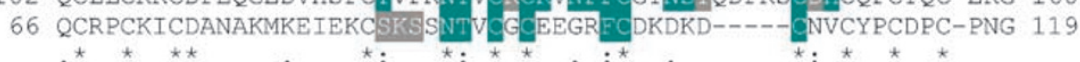

d Fas

DR4

CDEGHGLEVEINCTRTQNTKCR-CKPNFËCN-SWVDWCDPCTK-CE-HGIIKECTLTSN 162

170 CKSD-EEERSP-CTTTRNIACQ-CKPETT ERNDNSA-EMCRKCSRGCP-RGMVKVKDCTPW 224

119 CDSG-EVELSP-CTTTRNTVCQ-CEEGT FREEDSP-EMCRKCRTGCP-RGMVKVGDCTPW 173

DR5

TNER-1

NGFR

149 CLNG-TVHLS--CQEKQNTVCT-CHAGFGLRE----NECVSCSN-CK-KSLECTKLCLPQ 198

128 CEAGSGLVES--CQDKQNTVCEECPDGTYSDEANHV PCLPCTV-CE-DTERQLRECTRW 183

Death

receptor

DR3

CD40

TNFR-2

144 CGALHRHTRLL-CSRR-DIDCGT LLP GFYEH----DGCVSCPTSTLGSCPERCAAVCGW 197 (

members

$\operatorname{LT} \beta-\mathrm{R}$

125 CSPGFGVK-QI-AIGVSDIICEP PVEFESNVSSAF KCHPWTS-CETKDLVVQQAGTNK 181

143 CRPGFGVA-RP-GTETSDVVCKP AAPGT SNTTSSTEICRPHQI-CNVVAIPGNASMDAV 199

148 CPPGTEAELKD-EVGKGNNHGVPGKAGHENTSSPSARCQPHTR-CENQGLVEAAPGTAQ 205

e

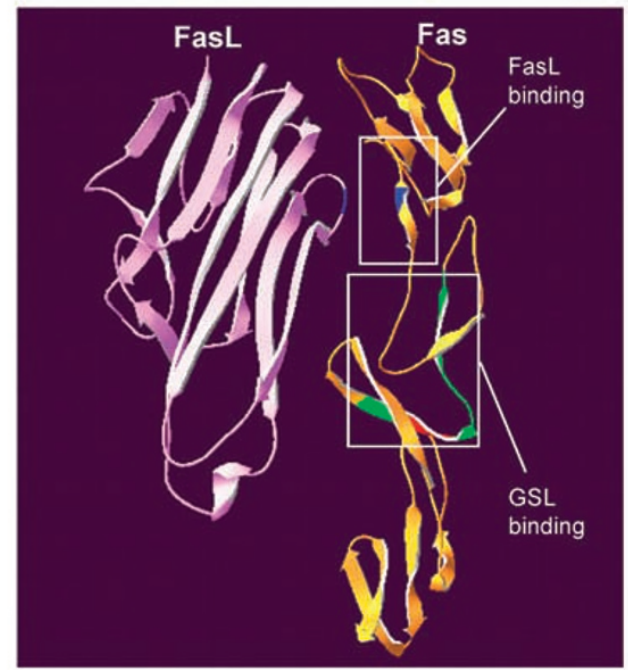


Fluorescence Correlation Spectroscopy (FCS). Through FCS diffusion law, we obtained the $y$-intercept $\left(t_{0}\right)$, indicative of the molecular confinement mode, and the effective diffusion coefficient $\left(D_{\text {eff }}\right)$, representing the long-range diffusion, of the proteins. ${ }^{17,18}$ The positive confinement times $\left(t_{0}\right)$ of both the hFas.GFP and the hFas.F134A.GFP (Figure 5c) indicated that both molecules were microdomain-confined, unlike molecules dislocated from the rafts which exhibit $t_{0}$ approaching zero. ${ }^{17-19}$ However, a drastically reduced $D_{\text {eff }}$ of hFas. F134A.GFP $\left(0.58 \mu \mathrm{m}^{2} / \mathrm{s}\right.$ versus $D_{\text {eff }}$ of $1.18 \mu \mathrm{m}^{2} / \mathrm{s}$ for hFas.GFP) indicates a more strongly impeded long-range diffusion of hFas134A-GFP which may be attributed to various factors including molecular complex formation (oligomerization-induced trapping model). ${ }^{20}$

Our complementing biochemical and biophysical data demonstrate that the GBM participates in the membrane dynamics of Fas. The microdomain-confined behaviour of GBM-defective Fas shown by FCS supports the biochemical observation that it remained localized in the DRM. Also, the possibility that the molecular complex formation may have caused the impeded diffusion behaviour of the GBM-defective Fas on the plasma membrane (reduced $D_{\text {eff }}$ as shown by FCS) is supported by the biochemical results showing that the GBM mutation favoured the higher order oligomer formation (Figure $5 a$ and b) and that the DRM-residing GBM-defective Fas (mostly higher order oligomers) represented a substantial proportion of cell surface Fas (unpublished data).

The Y130A mutation inhibits the DISC formation but not the Fas-ezrin association and Fas internalization. We further investigated the Fas-ezrin association, receptor internalization, and the DISC formation, the prerequisites for Fas-mediated cell death following raft targeting by Fas palmitoylation. ${ }^{6}$ Notably, although the Y130A mutation resulted in the markedly defective DISC formation, as shown by the loss of the FADD and caspase 8 recruitment upon the activation with FasL, it did not inhibit the Fas-ezrin association (Figure 6a) nor the receptor internalization (Figure 6b)

The internalization of the wild type Fas, but not the GBM-defective Fas, is clathrin-dependent. As GBMdefective cells had a blockage in DISC formation and cell death whereas their Fas-ezrin association and receptor internalization remained efficient and the non-death signals were enhanced, we hypothesized that GBM may exert its role in selective internalization of the receptor and thus the signalling modes. As clathrin-mediated endocytosis (CME) has been shown involved in Fas-mediated apoptosis, ${ }^{5}$ we investigated the GBM's involvement in the clathrin-mediated Fas internalization.

Monodansylcadaverine (MDC), a CME inhibitor that stabilizes clathrin-coated vesicle and prevents uncoating, is presumed to inhibit CME by reducing the level of free clathrin to assemble at the plasma membrane. ${ }^{21}$ MDC treatment caused a dose-dependent internalization delay of the FasLactivated mFas (Figure 7a), whereas the internalization of mFas. Y130A remained unaffected (Figure $7 \mathrm{~b}$ ). The treatment with dynasore, ${ }^{22}$ a potent inhibitor of dynamin, significantly hindered the internalization of mFas but not that of mFas. Y130A (Figure 7c and d). Likewise, reducing clathrin heavy chain $(\mathrm{CHC}$ ) expression using shRNAi (Figure $7 \mathrm{~g}$ ), perturbed only the internalization of $\mathrm{mFas}$ (Figure 7e) but not that of the mFas.Y130A (Figure 7f).

Notably, these treatments only hampered the Fas internalization in L1210.mFas cells but not in the L1210.mFas. Y130A cells despite the equivalent inhibition of the internalization of transferrin, a CME marker, in both cell lines (Supplementary Figure S4A-F). Also CHC shRNAi inhibited the Fas-mediated cell death in L1210.mFas cells (Figure 7h), confirming the importance of $\mathrm{CHC}$ in the Fas internalization and cell death as shown in a human context. ${ }^{5}$

These results suggest that the cell death signalling of Fas is connected to its internalization through CME. And by disrupting the GBM, Fas can be internalized, instead, through an alternative clathrin-independent pathway, which allow the surge of certain non-death signals.

Wild type Fas preferentially associates with clathrin assembly proteins. Immunofluorescence showed that Fas-FasL complex associated with $\mathrm{CHC}$ and CALM more in L1210.mFas cells (Figure 8a and $c$ ) than in the L1210.mFas.Y130A cells (Figure $8 b$ and $d$ ). This was confirmed by quantitative colocalization analyses (Figure $8 \mathrm{e}$ and f). Conversely, coimmunoprocipitation showed that flotillin-1 and flotillin-2 (Figure $8 \mathrm{~g}$ ), which have been implicated in the clathrin-independent internalization ${ }^{4,23}$ associated more with mFas.Y130A than mFas. These results suggest that the GBM disruption may shift the internalization preference of activated Fas from CME toward a clathrin-independent pathway.

The internalization of mFas.Y130A is independent of cholesterol-dependent rafts but dependent on ezrin. To understand the alternative internalization due to GBM defect,

Figure 1 Fas extracellular domain contains glycosphingolipid-binding motif (GBM). (a) Location of the putative GBM in Fas (CRD, cysteine-rich domain; TM, transmembrane; DD, death domain) and multiple amino acid sequence alignment of the putative GBM of murine Fas (mFas) and human Fas (hFas), and HIV-1 gp120 V3 loop (V3) using ClustralW (http://www.ebi.ac.uk/Tools/clustalw/) followed by manual adjustment (green-shaded, conserved amino acids; gray-shaded, conserved substitutions; numbers, positions of amino acids; '*', residues absolutely conserved in all aligned sequences; ':', conserved substitutions based on physio-chemical properties have been observed; ' 's semi-conserved substitutions have been observed, (b) Simulation showing the superposition of Fas GBM with HIV-1 gp120 V3 loop (blue, Fas GBM; red, V3 loop), (c) Multiple sequence alignment of amino acid stretches covering the putative GBM of Fas in various animal species, with CRD2 and CRD3 portions of the hFas indicated above the alignment. (d) Multiple sequence alignment of amino acid stretches covering the putative GBM of hFas and the corresponding amino-acid sequences of some other TNFR superfamily members. Accession numbers of aligned proteins are listed in Supplementary Information. (e) Structural model of the complex between extracellular domain of human Fas (gold) and human FasL (pink) generated using Swiss Model indicating Fas-FasL interaction site with the major contributing residues R102 of Fas and Y218 of FasL (blue); and the GBM forming a loop (green) with the F133 and F134 at the turn (red) 

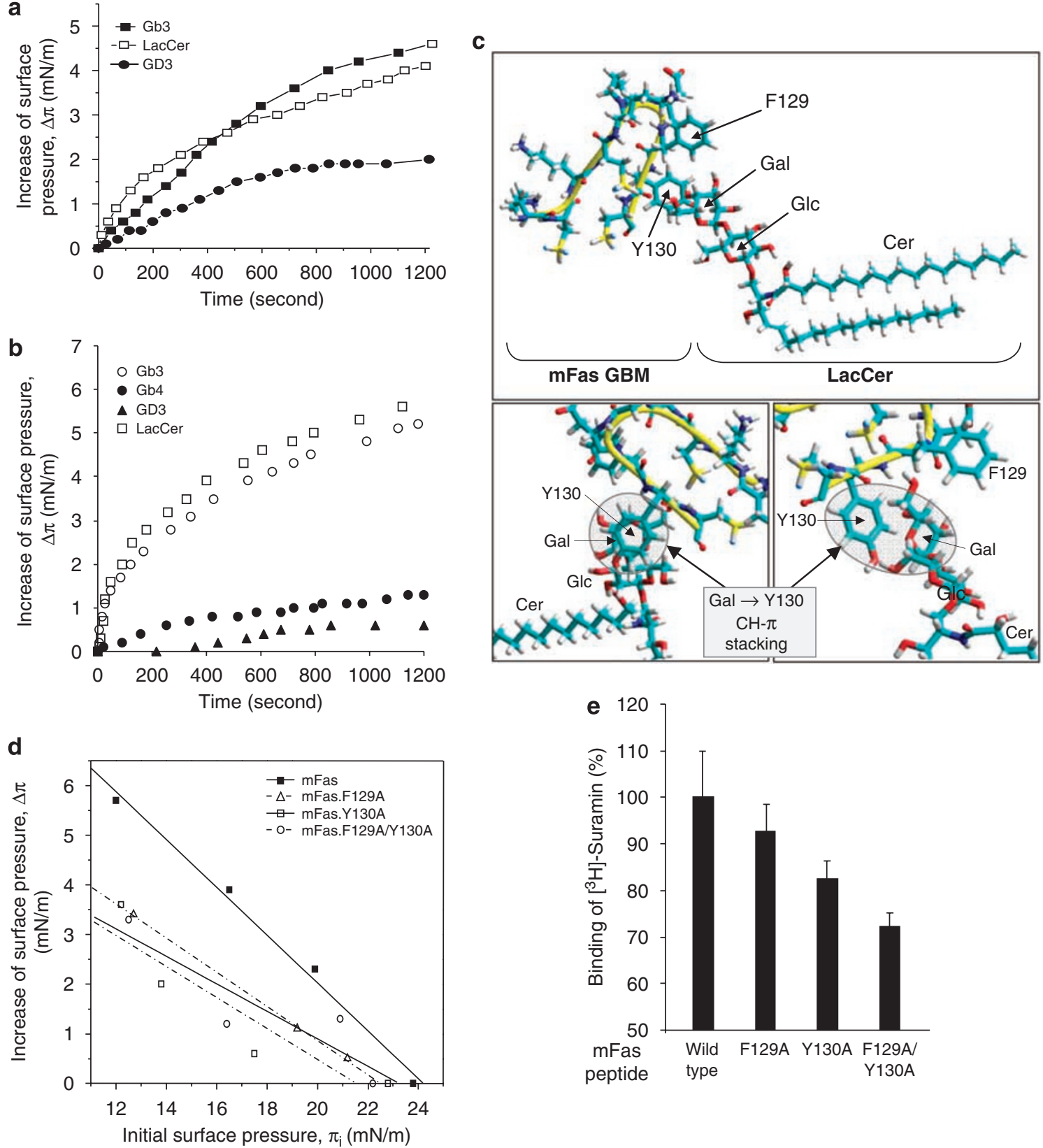

Figure 2 Fas GBM selectively binds to certain glycosphingolipids and its aromatic residues were essential for this binding. Surface pressure isotherms measured by Langmuir film balance technology represent the time-dependent surface pressure increase $(\Delta \pi)$ of glycosphingolipid monolayers. The interaction intensity is correlated to the magnitude of $\Delta \pi$ because of the penetration of the lipid monolayers by the interacting protein (see Supplementary Information). (a) $\Delta \pi$ induced by the recombinant extracellular domain of Fas protein $\left(2.5 \mathrm{mM}\right.$, initial pressure $\left(\pi_{i}\right)$ of $\left.12 \mathrm{mN} / \mathrm{m}\right)$. (b) $\Delta \pi$ induced by the synthetic peptide derived from mFas GBM $\left({ }^{122} \mathrm{KCKCKPDFYCDSPGCEH}^{138}, \pi_{\mathrm{i}}=10 \mathrm{mN} / \mathrm{m}\right)$. (c) Molecular model of interaction between the GBM of mFas and LacCer showing the GBM in the hairpin structure interacting with LacCer (upper panel; Gal, galactose; Glc, glucose; Cer, ceramide backbone) and the 'stacking' between the residue Y130 against the galactose ring of LacCer, rendering the $\mathrm{CH}-\pi$ interaction (lower panels). (d) The reduction in the $\Delta \pi$ after injection of mFas GBM peptide (or mutant mFas GBM peptides with indicated amino-acid substitutions) under LacCer monolayer, as the initial surface pressures $\left(\pi_{i}\right)$ increase indicates the specific peptide-LacCer binding, with a drastic reduction in the binding efficiency with the mutant GBM peptides (represented by the drop of $\Delta \pi$ at all $\pi_{i}$ examined). (e) [ ${ }^{3} \mathrm{H}$ ]-Suramin was incubated in 96 -well multiplates coated with GBM peptides (wild type or indicated mutant at $100 \mathrm{ng} / \mathrm{well}$ ) and the bound radioactivity was measured with scintillation counter. Data are shown as mean \pm S.D.

we investigated the requirement of cholesterol-dependent rafts (chol-rafts). Following cholesterol depletion through cholesterol oxidase (CO) treatment, Fas internalization in L1210.mFas cells was significantly delayed whereas that of the L1210.mFas. Y130A cells remained unaffected (Figure $9 a$ and b). The treatment did not interfere with the transferrin internalization, suggesting that
CO did not interfere with CME (Supplementary Figure S4). This chol-raft-independent internalization of mFas.Y130A was also confirmed with metabolic cholesterol depletion by lovastatin treatment (not shown).

Fas association with ezrin, a membrane protein-actin cytoskeleton linker is required for the Fas internalization and 

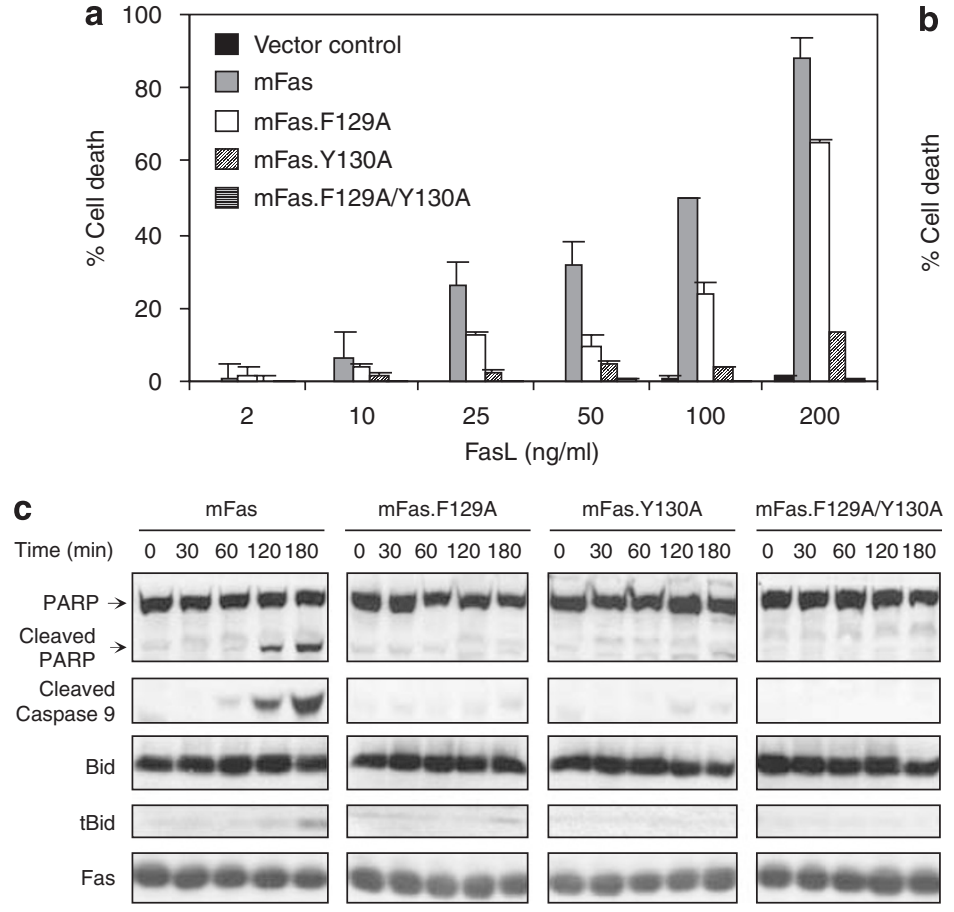

Figure 3 The glycosphingolipid-binding motif is essential for cell death signalling of Fas. L12.10.3 cells stably expressing the indicated mFas protein were incubated with the designated amounts of rhFasL crosslinked with $\mathrm{M} 2$ for $4 \mathrm{~h}$, (a); or with $50 \mathrm{ng} / \mathrm{ml}$ rhFasL crosslinked with $\mathrm{M} 2$ for the specified time, (b); before assessing for cell death by FACS; or stimulated with $50 \mathrm{ng} / \mathrm{ml}$ of rhFasL crosslinked with M2 for the specified time before lysis and heating at $95^{\circ} \mathrm{C}$ in Laemli buffer and subsequently SDS-PAGE and immunoblotting with indicated antibodies, (c); or subjected to coculture with J16/Rapo-FasL (killer) or J16/Rapo (control) cells for $18 \mathrm{~h}$ before cell death assessment by FACS (d). Data are shown as mean \pm S.D. The apoptosis inhibition by the GBM mutations was specific for Fas-mediated cell death as the cell death by other apoptotic stimuli, for example, staurosporin, was not inhibited (data not shown)

cell death signalling. ${ }^{6,24}$ Similar to what we have reported for $\mathrm{mFas}^{6}$ (and data not shown), ezrin shRNAi delayed mFas. Y130A internalization (Figure $9 \mathrm{c}$ and $\mathrm{d}$ ). Moreover, the overexpression of the phospho-Y353-deficient ezrin also hindered mFas.Y130A internalization (Figure 9e and f). As ezrin phosphorylation at Y353 is important for its activation, linkage to membrane proteins, and signal transduction, ${ }^{25,26}$ these results suggest that the alternative chol-raft- and clathrin-independent internalization process of mFas.Y130A requires ezrin's functions.

\section{Discussion}

We present for the first time the multiple internalization pathways of the death receptor, Fas, and the protein/lipidbased mechanism responsible for its selective post-ligand internalization and the subsequent signalling mode. We identified an extracellular GBM of Fas as one of the requirements for the ligand-activated receptor internalization through CME, previously reported essential for the efficient apoptotic signal. The loss of function of GBM drives the receptor to the clathrin-independent internalization route, preventing its death signal and potentiating its non-death signals.

The genetic proofs of the importance of CME in the Fas signalling was first demonstrated in a human $B$ cell line where $\mathrm{CHC}$ and AP-2 siRNA inhibited Fas internalization and cell death but spared the Fas-mediated p44/42 MAPK activation. ${ }^{5}$ Although we also observed through RNAi and pharmacological approaches that the inhibition of the CME hindered the internalization of the mFas, and thus Fas-induced apoptosis, these inhibitory treatments could not inhibit the internalization of the GBM-defective Fas. This suggests the alternative internalization route(s) that Fas may take in certain circumstances, for example, upon the loss of proper glycosphingolipid interaction due to GBM defects, leading to a surge of non-apoptotic signalling as presented here.

During our study, we noted in all the CME inhibition treatments a systematically clear dose-dependent inhibition of Fas internalization at early activation time points followed by a dose-independent recovery of the Fas internalization at a longer activation time. Although it is possible that insufficient treatments caused the incomplete CME inhibition, other alternative internalization systems might have also rapidly compensated for the impaired CME in our cell system. Studies have shown that the compensatory clathrin-independent internalization can be upregulated upon the loss of $\mathrm{CME}{ }^{27}$ Indeed despite the Fas internalization recovery that occurred at a longer activation time point in CHC shRNA-transfected cells, the sensitivity toward Fas-mediated apoptosis was not recovered in these cells (Figure $7 \mathrm{~h}$ ). It is possible that the later internalization recovery might be through the alternative pathway which did not promote the cell death and that it was rather the $\mathrm{CME}$ that occurred very rapidly at the early 

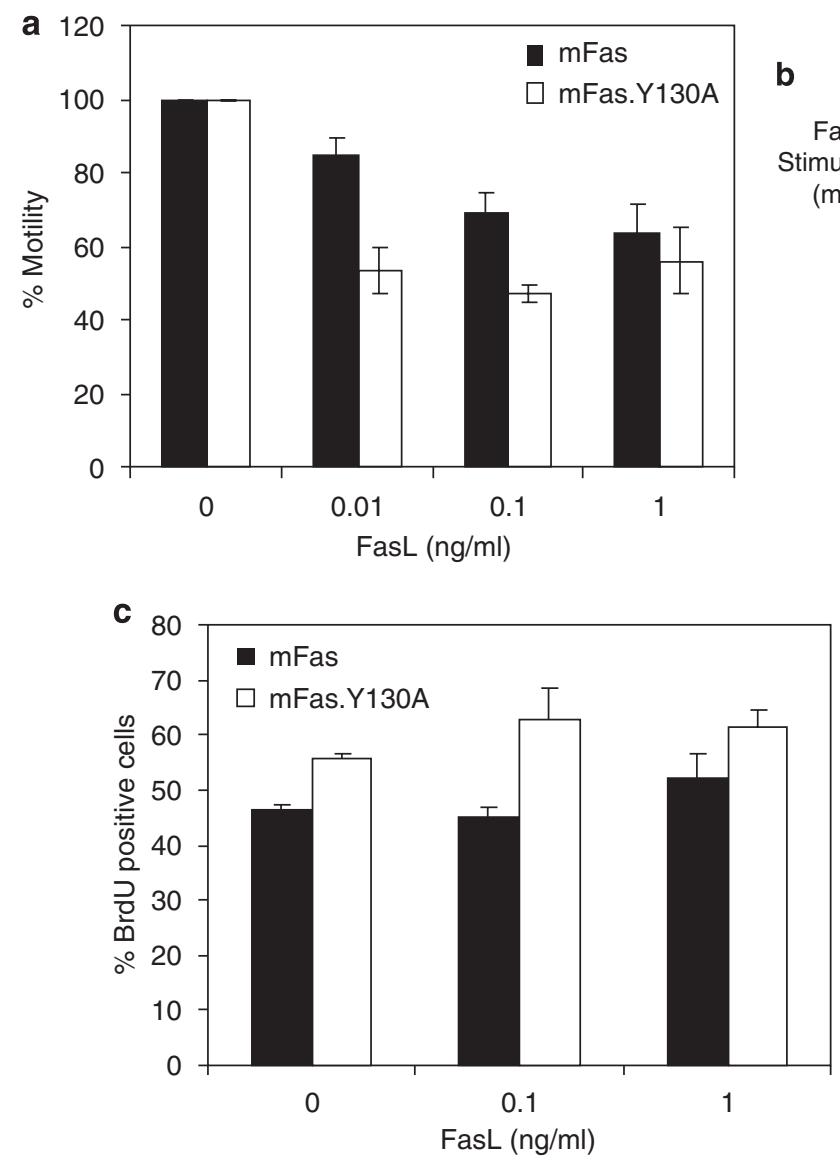

b

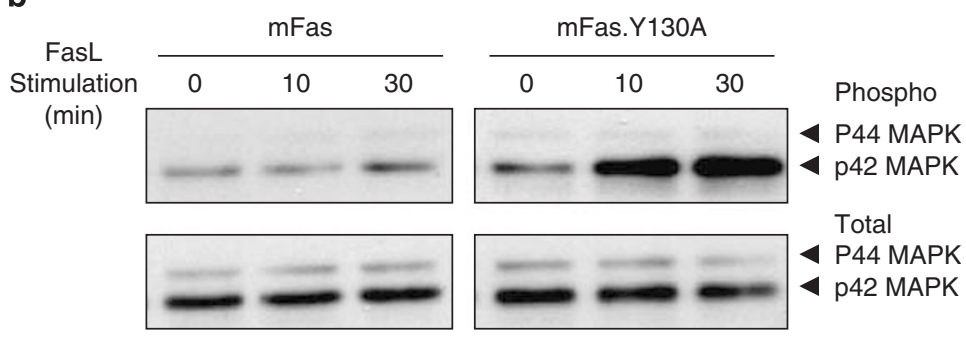

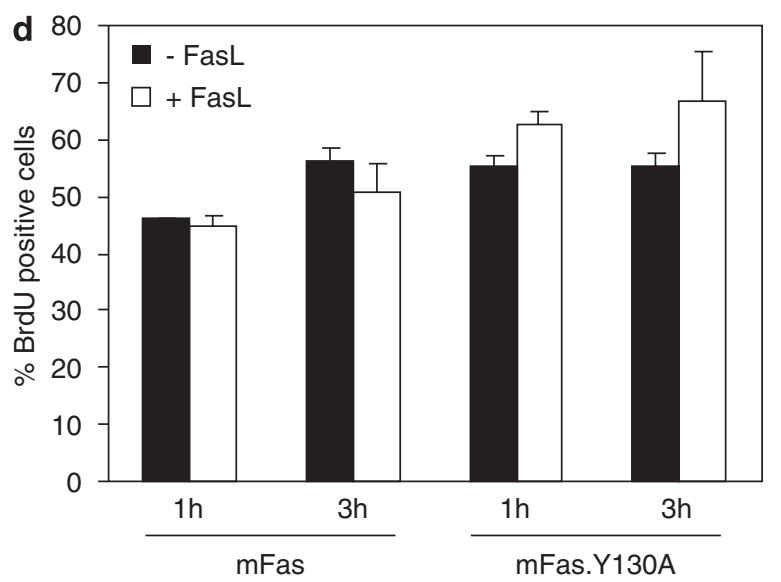

Figure 4 The glycosphingolipid-binding motif participates in the control of non-death signals of Fas. (a) Motility assay using Boyden chambers performed on L1210.mFas and L1210.mFas.Y130A cells in the presence of indicated amount of rhFasL crosslinked with M2. The motility of cells upon stimulation with rhFasL is shown relative to that of the unstimulated cells (represented as having the degree of motility of $100 \%$ ). (b) L1210.mFas and L1210.mFas.Y130A cells were incubated with $1 \mathrm{ng} / \mathrm{ml}$ of rhFasL crosslinked with M2 for an indicated time before the lysates were collected and subjected to SDS-PAGE and immunoblotting with indicated antibodies. BrdU proliferation assay was performed on L1210.mFas and L1210.mFas.Y130A cells stimulated with indicated amount of rhFasL crosslinked with M2 for $1 \mathrm{~h}$ (c), or with $0.1 \mathrm{ng} / \mathrm{ml}$ of rhFasL crosslinked with $\mathrm{M} 2$ for the indicated time (d). Degree of proliferation is represented by the percentage of cells that were BrdU-positive. Data are shown as mean \pm S.D.

activation point (which was inhibited in these cells) that accounted for most of the apoptotic signalling.

Our evidence suggests that the internalization of FasLactivated Fas involves the integrity of cholesterol-dependent rafts (chol-rafts) and CME. However, it is unclear whether chol-raft- and clathrin-mediated pathways operate mutually or in parallel in the internalization of activated Fas. Owing to reports that some prototypic receptors that are internalized by CME, for example, TfR and LDLR, are not enriched in rafts, ${ }^{28,29}$ it has been generally assumed that CME is separated from the raft-mediated internalization. However the possibility that the spatial organization of Fas in distinct microdomains may affect its CME is not as paradoxical as it may appear because the involvement of specialized microdomains in the assembly of CME components has been shown. In B cell lines, the BCR internalization requires the localization of clathrin to rafts, where it becomes phosphorylated. $^{30}$ The EGFR and CME components, for example, clathrin, AP2, and Eps15, are recruited to cholesteroldependent DRM upon stimulation with EGF and the EGFRinternalizing clathrin-coated pits form preferentially from GM1-rich plasma membrane regions. ${ }^{31}$ Also, the anthrax toxin-induced relocalization of anthrax toxin receptor to the chol-rafts is a prerequisite for its Cbl-mediated ubiquitination and $\mathrm{CME}^{32,33}$

Our biochemical and biophysical analyses indicate GBM's role in the heterogeneous microdomains localization, multimolecular complex formation, and diffusion of Fas (Figure 5). Although FCS showed the similar microdomain-confined behaviour for wild type and GBM-defective Fas, the biochemical results not only supported the FCS observations by showing that both wild type and GBM-defective Fas partitioned into DRM but also revealed that the GBM-defective Fas preferentially resided in the DRM of lower density than what observed for DRM-residing wild type Fas. Moreover, unlike wild type Fas, most of GBM-defective Fas in DRM formed high-order oligomers, and thus providing an explanation for its dampened diffusion shown by FCS.

These results suggest the GBM's glycosphingolipid-interacting functions in the organization of Fas in membrane microdomains. The functional GBM allows Fas to localize in a certain class of microdomains, which may favour its interactions with the CME machinery. The loss of interactions with certain glycosphingolipids due to GBM defect may cause Fas 
a
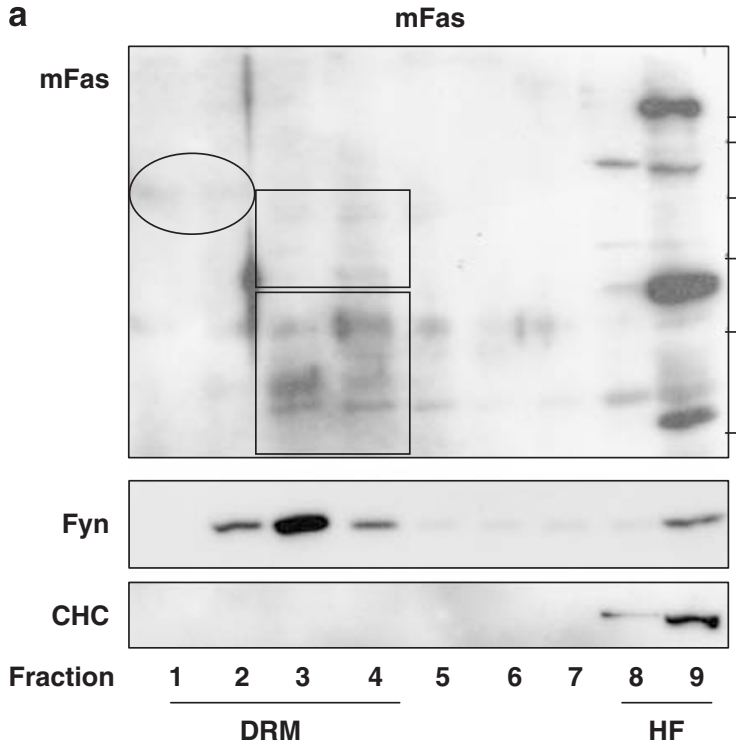

mFas.Y130A
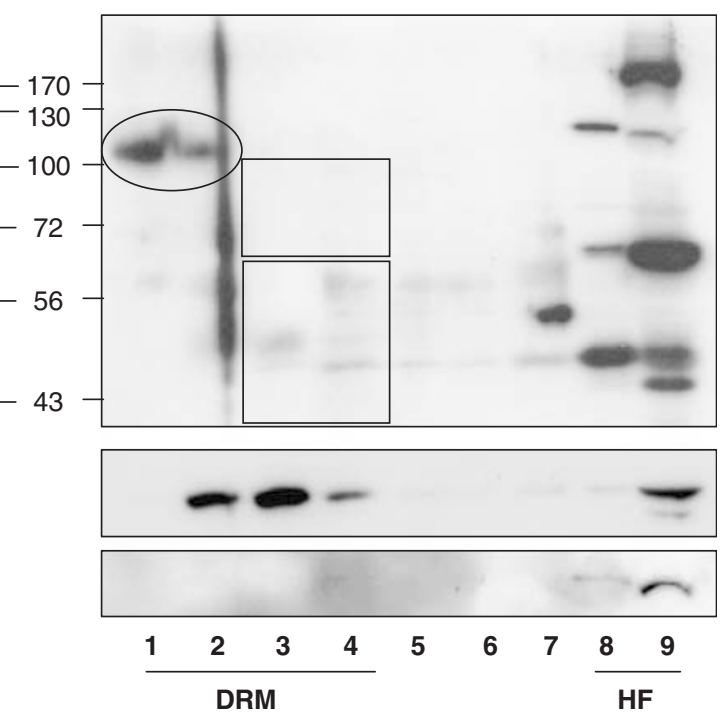
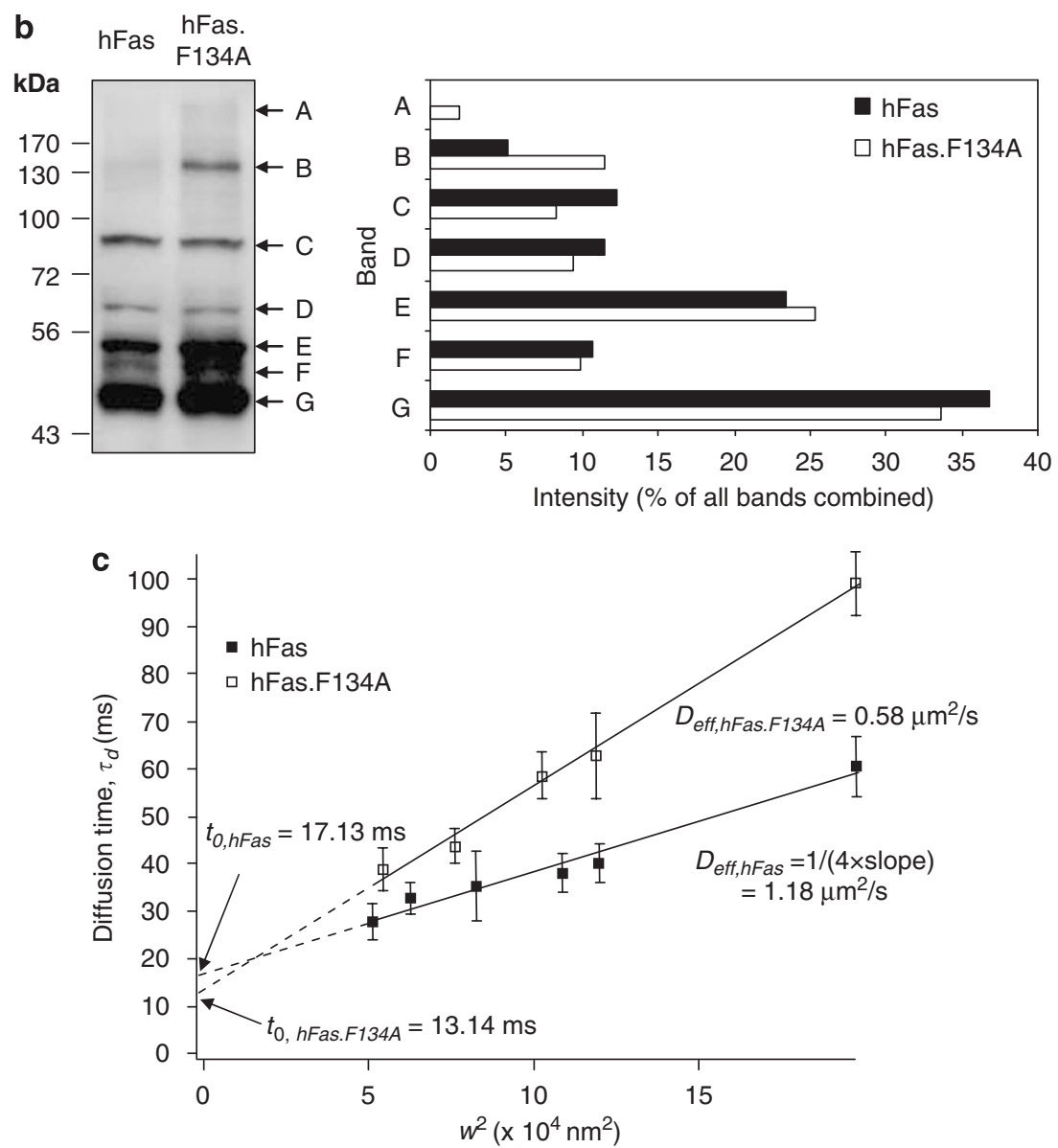

Figure 5 The glycosphingolipid-binding motif of Fas plays a part in the membrane dynamics of the receptor but not in the raft targeting. (a) L1210.mFas and L1210.mFas.Y130A cells $\left(3 \times 10^{7}\right)$ were subjected to low density, detergent-resistant membrane microdomain (DRM) preparation. DRMs are localized in fractions 1-4. HF denotes high-density fractions where detergent-solubilized proteins or those that may remain with high-density microdomains are localized. Fyn and $\mathrm{CHC}$ serve as markers for DRM and HF, respectively. (b) CAL51 cells were transiently transfected with hFas or hFas.F134A constructs by calcium phosphate precipitation. The lysates were collected $24 \mathrm{~h}$ after transfection and subjected to SDS-PAGE and immunoblotting with anti-hFas antibody (left panel). The intensity of the bands indicated by letters on the immunoblot was quantified using ImageJ $1.37 \mathrm{l}$ and presented in the right panel as percentage of total intensity of all bands. (c) Diffusion behaviour of hFas-GFP and hFas.F134A-GFP transiently expressed in COS-7 cells and analyzed by FCS as described. ${ }^{6}$ Diffusion time $\left(\tau_{d}\right)$ was plotted as a function of illuminated spot size, that is, the squared radius $\left(w^{2}\right)$. Based on the FCS diffusion law, $\tau_{\mathrm{d}}=t_{0}+\left(w^{2}\right) /\left(4 D_{\text {eff }}\right)$, the confinement time $\left(t_{0}\right)$ and the effective diffusion coefficient $\left(D_{\text {eff }}\right)$ were deduced from the intercept and the slope of the curve, respectively ${ }^{17}$ 

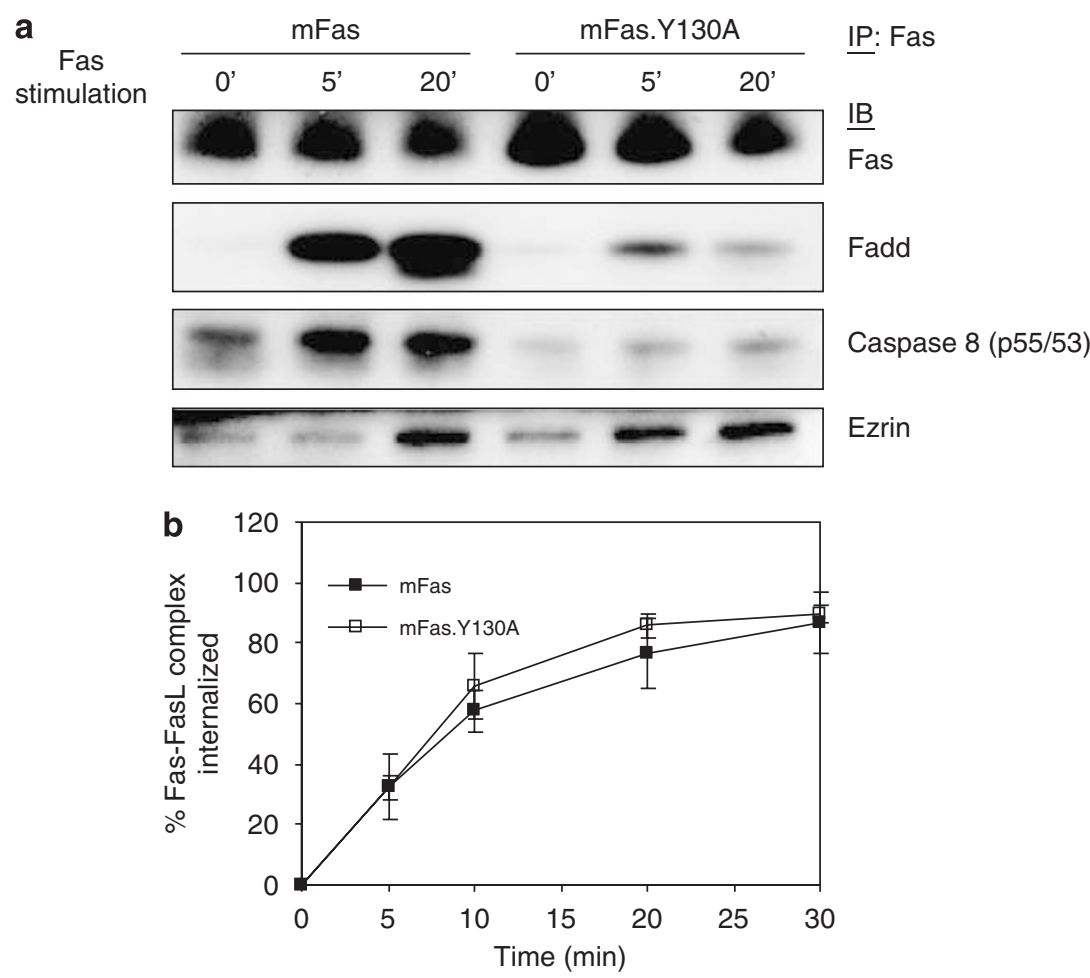

Figure 6 The lack of the critical tyrosine residue in the glycosphingolipid-binding motif inhibits the DISC formation but not ezrin association and the internalization of Fas after FasL engagement. (a) L1210.mFas and L1210.mFas.Y130A cells $\left(3 \times 10^{7}\right)$, unstimulated or stimulated with $100 \mathrm{ng} / \mathrm{ml}$ Flag-rhFasL plus $1 \mu \mathrm{g} / \mathrm{ml}$ M2 at $37^{\circ} \mathrm{C}$ for the indicated time, were subjected to DISC isolation by Fas coimmunoprecipitation. The immunoprecipitates were subjected to SDS-PAGE and immunoblotting (IB) with indicated antibodies. (b) L1210.mFas and L1210.mFas.Y130A cells were subjected to Fas internalization assay upon synchronized stimulation (data shown as mean \pm S.D.)

to localize in a different subclass of microdomains that may favour a stronger oligomerization and the alternative internalization mechanism that is independent of clathrin and cholrafts. This may then cause Fas to switch its function from cell death to non-death mode.

The exact internalization mechanism of the GBM-defective Fas remains unclear. Our findings indicate that: (1) the GBM-defective Fas can be internalized independently of cholesterol-dependent rafts (Figure 9b), and (2) it has an increased association with flotillins (Figure $8 \mathrm{~g}$ ). Notably, flotillins have been shown enriched in the detergent-resistant membrane microdomains that are resistant to cholesterol depletion and thus have been regarded as proteins that define a subset of DRM. ${ }^{34}$ Moreover, flotillin-1 has recently been shown as one of the determinants of clathrin-independent endocytosis. ${ }^{23}$ Overall, one may speculate different internalization scenarios that the GBM defect may render. One possibility is that GBM defect leads to the relocalization of Fas to the subtype of the microdomains that have a different glycosphingolipid profile that may resist cholesterol depletion and are rich in flotillins, which may in turn mediate the clathrinindependent internalization. Yet, it is also possible that the internalization is through the surface pool of GBM-defective Fas that is neither raft- nor flotillin-associated. These hypotheses require further investigations.

An additional clue to the complex internalization mechanisms of Fas is given by our observation that although the internalization processes of the wild type and GBM-defective Fas differ in the requirement for chol-rafts and CME, they shared the requirement for ezrin. This implicates the manner of Fas-actin cytoskeleton linkage as one of the mechanisms underlying both internalization pathways. Although the mechanisms of Fas internalization and its signalling remain to be explored, our studies have revealed different internalization behaviours between wild type and GBM-defective Fas which culminate in different cellular outcomes.

It is noteworthy that although the critical aromatic residue of the Fas GBM appears conserved among vertebrates, the high sequence similarity of the entire GBM is only observed among mammalian species (Figure 1c). Possibly, there is a selection pressure for this motif in mammals where its functions may have diverged from that of the non-mammalian species along the course of evolution. This sequence conservation of the GBM is not a general feature of the CRDs as the sequence identity in Fas GBM is significantly higher than in the sequence encompassing the three CRDs (52\% sequence identity within the GBM versus $\sim 36 \%$ for the entire Fas CRDs among mammalian species, Supplementary Figure S1D). Moreover, although the amino-acid sequences in the extracellular domains of the TNFR superfamily members vary considerably, an appreciable similarity is observed around the stretch corresponding to Fas GBM, particularly among death receptors. Possibly, this motif may participate in the multi-signal regulations of other TNFR superfamily members as well.

Overall, this study demonstrates the significance of proteinlipid interactions in the membrane receptor signalling regulation, which may explain adaptable cellular responses upon the 

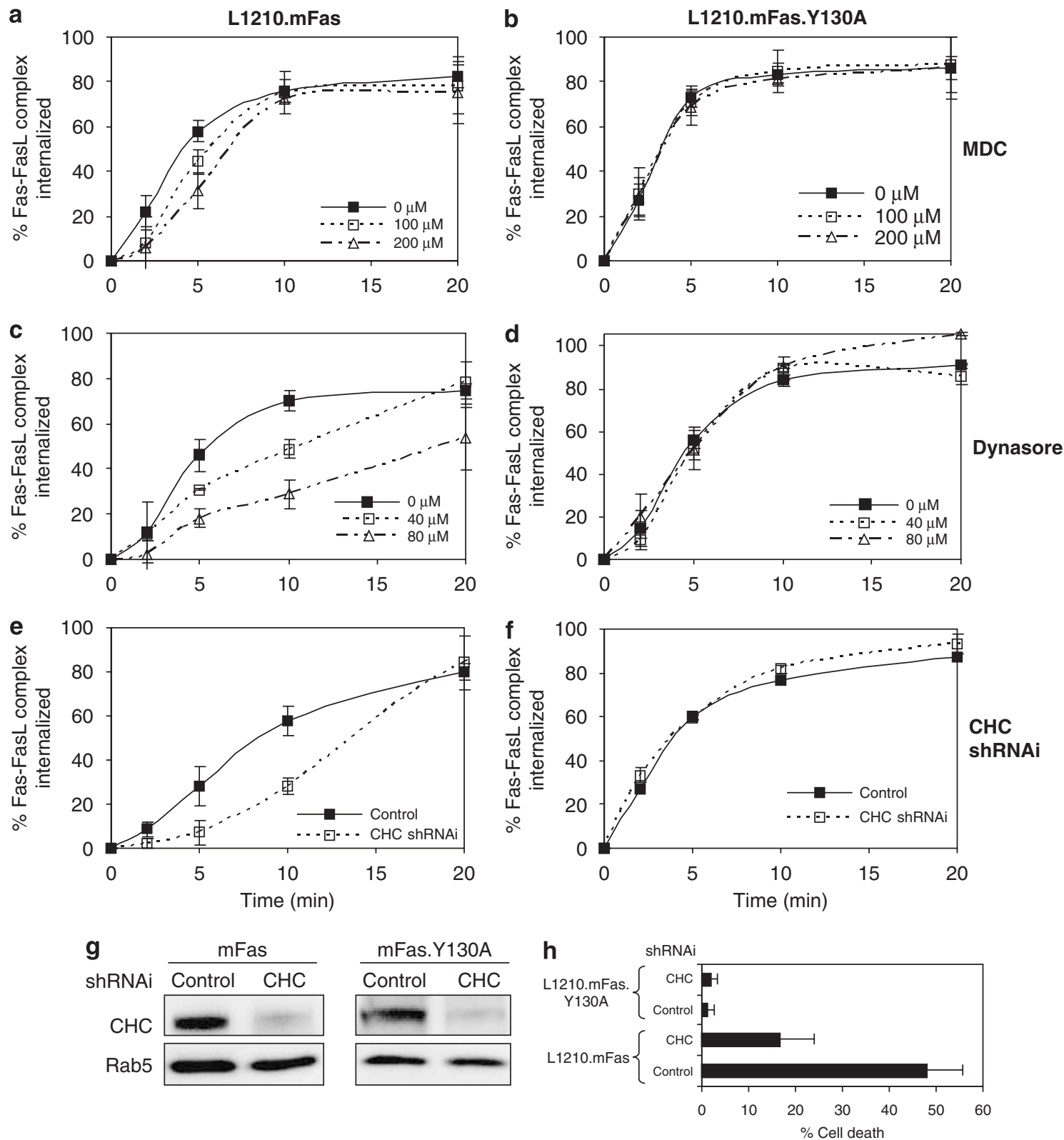

Figure 7 The internalization of wild type Fas after activation is clathrin-dependent whereas that of the glycosphingolipid-binding mutant Fas is not. L1210.Fas and L1210.mFas.Y130A cells were untreated or treated with indicated amounts of MDC or dynasore for $30 \mathrm{~min}$ at $37^{\circ} \mathrm{C}$ or stably transfected with indicated shRNAi before subjecting to Fas internalization (a-f). L1210.mFas and L1210.mFas.Y130A cells expressing indicated shRNAi were lysed in Laemli buffer at $95^{\circ} \mathrm{C}$ and subjected to SDSPAGE and immunoblotting with indicated antibodies $(\mathbf{g})$ or were incubated with $50 \mathrm{ng} / \mathrm{ml}$ rhFasL crosslinked with M2 for $6 \mathrm{~h}$ and analyzed for cell death (h). Data are shown as mean \pm S.D.

activation of various versatile receptors in different tissues and conditions. A better understanding in this issue can shed light into the connection between perturbation of lipid profiles observed in certain diseases and the shift in signalling balance of receptors, including Fas, which can contribute to clinical manifestations, for example, of neurodegenerative diseases and cancers.

\section{Materials and Methods}

Constructs and reagents. Constructs, antibodies, and reagents used are listed in Supplementary Information)
Cell transfections. Transient transfections in COS-7 cells and stable transfections with mFas, mFas.F129A, mFas.Y130A, and mFas.F129A/Y130A constructs in Fas-deficient L12.10.3 cells to obtain L1210.mFas, L1210.mFas.F129A, L1210.mFas.Y130A, and L1210.mFas.F129A/Y130A cells with equivalent Fas expression (Supplementary Figure S2) were performed as described. ${ }^{6}$ L1210.mFas or L1210.mFas.Y130A cells expressing short hairpin RNAi (shRNAi) of CHC, ezrin, or control were established by transfection with pTER plasmids containing shRNAi sequence of mouse CHC (See Supplementary Information), mouse Ezrin, or SIMA (control), ${ }^{6}$ respectively, and selected for stable cells with Zeocin resistance.

Protein modelling. The Fas-FasL complex model was generated using the Swiss Model ${ }^{11,35}$ with 3-dimensional structures of $\mathrm{TNF}_{\alpha}, \mathrm{TNF}_{\beta}$, as templates for 
a
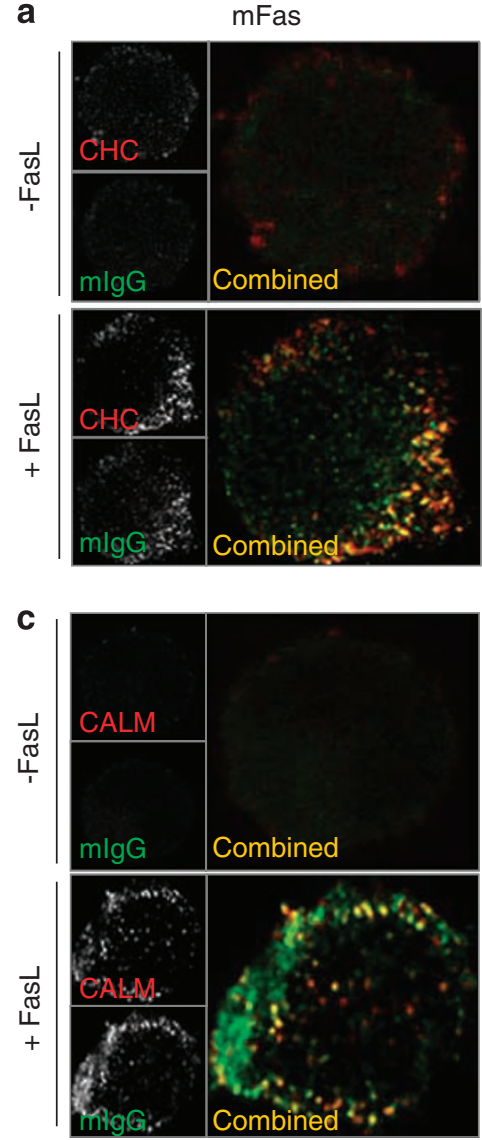

b
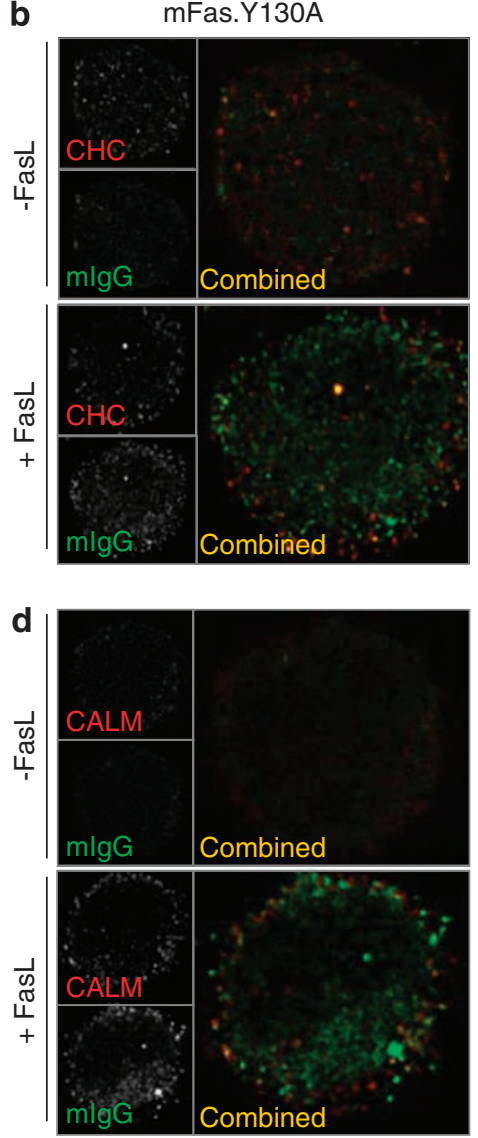
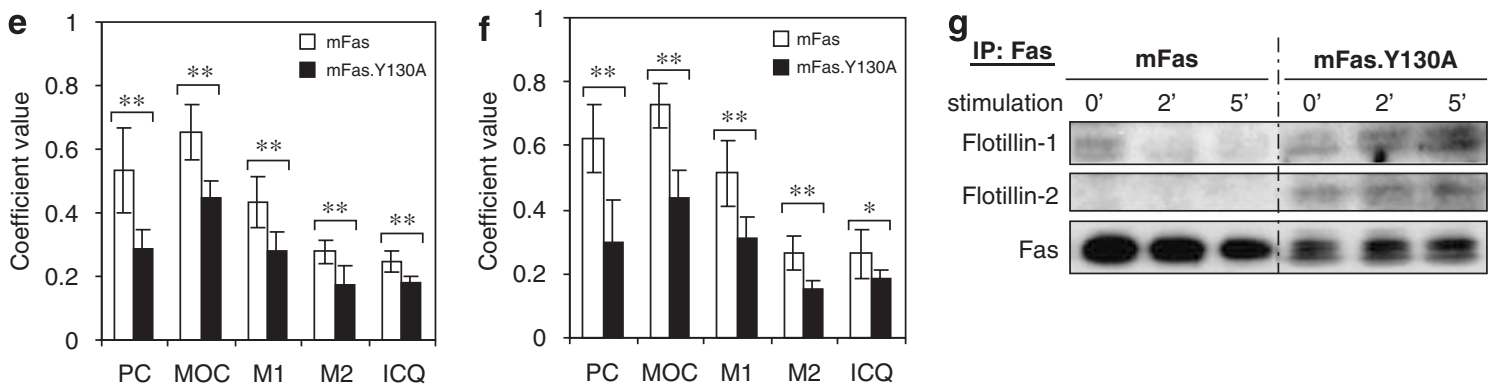

Figure 8 GBM defect decreases Fas association with clathrin-assembly proteins. L1210.mFas (a and $\mathbf{c}$ ) and L1210.mFas.Y130A (b and $\mathbf{d})$ were incubated for 2 min at $37^{\circ} \mathrm{C}$ in the presence or absence of $200 \mathrm{ng} / \mathrm{ml} \mathrm{FasL}$ and $1 \mu \mathrm{g} / \mathrm{ml} \mathrm{M2}$, immunostained for CHC or CALM with corresponding antibodies as well as fluorescence-conjugated antimouse IgG (mlgG) to detect Fas-FasL-M2 complex before the observation with confocal microscopy. Images are representative of cells typically observed from two independent experiments. Confocal images were also subjected to quantitative colocalization analysis using JACoP colocalization analysis plug-in in ImageJ. Colocalization values obtained from various approaches listed in the Materials and Methods section were shown for FasL-stimulated cells that where subjected to costaining for FasL/Fas complexes and CHC (e) or CALM (f) (values shown as mean \pm S.D., statistical significance were analysed using student's $t$-test, ${ }^{* \star} P<0.01,{ }^{*} P<0.05, n=4$ ). For each image analysed, the Costes' $P$-value from Costes' randomized analysis was $100 \%$, indicating high probability that the colocalization observed was not the colocalization of pixels due to chance (see Materials and Methods). (g) L1210.mFas and L1210.mFas.Y130A cells $\left(3 \times 10^{7}\right)$ were incubated without or with $200 \mathrm{ng} / \mathrm{ml} \mathrm{FasL} \mathrm{plus} 1 \mu \mathrm{g} / \mathrm{ml} \mathrm{M} 2$ at $37^{\circ} \mathrm{C}$ for the indicated time and then subjected to Fas immunoprecipitation (IP) followed by SDS-PAGE and immunoblotting (IB) with indicated antibodies

FasL and TNFR1 for Fas (PDB entries 1TNF chain a, b, c; 1TNR chain A, and 1TNR chain $R$, respectively). The Fas-FasL complex structure was generated based on the crystal structure of TNFR1-TNFb complex ${ }^{36}$ and view with Swiss PDB viewer v.3.7. The other models were generated with the Hyperchem software as described. $^{37,38}$

Lipid monolayer surface pressure measurements by Langmuir film balance technique. An automated microtensiometer was used to record the pressure-area compression isotherms and the interaction kinetics between peptides and indicated monolayer lipid film as described. ${ }^{9}$ Synthetic Fas peptides or rED-Fas were injected under a monomolecular lipid film and the pressure increases were recorded until reaching the equilibrium. See Supplementary Information for more details.

Solid-phase $\left[{ }^{3} \mathrm{H}\right]$-suramin-binding assay. The assay was performed as described. ${ }^{16}$ Fas peptides were coated in 96-well multiplates. After washing and subsequent blocking with $1 \%$ gelatin, the plates were incubated $\left(2 \mathrm{~h}, 37^{\circ} \mathrm{C}\right)$ with 

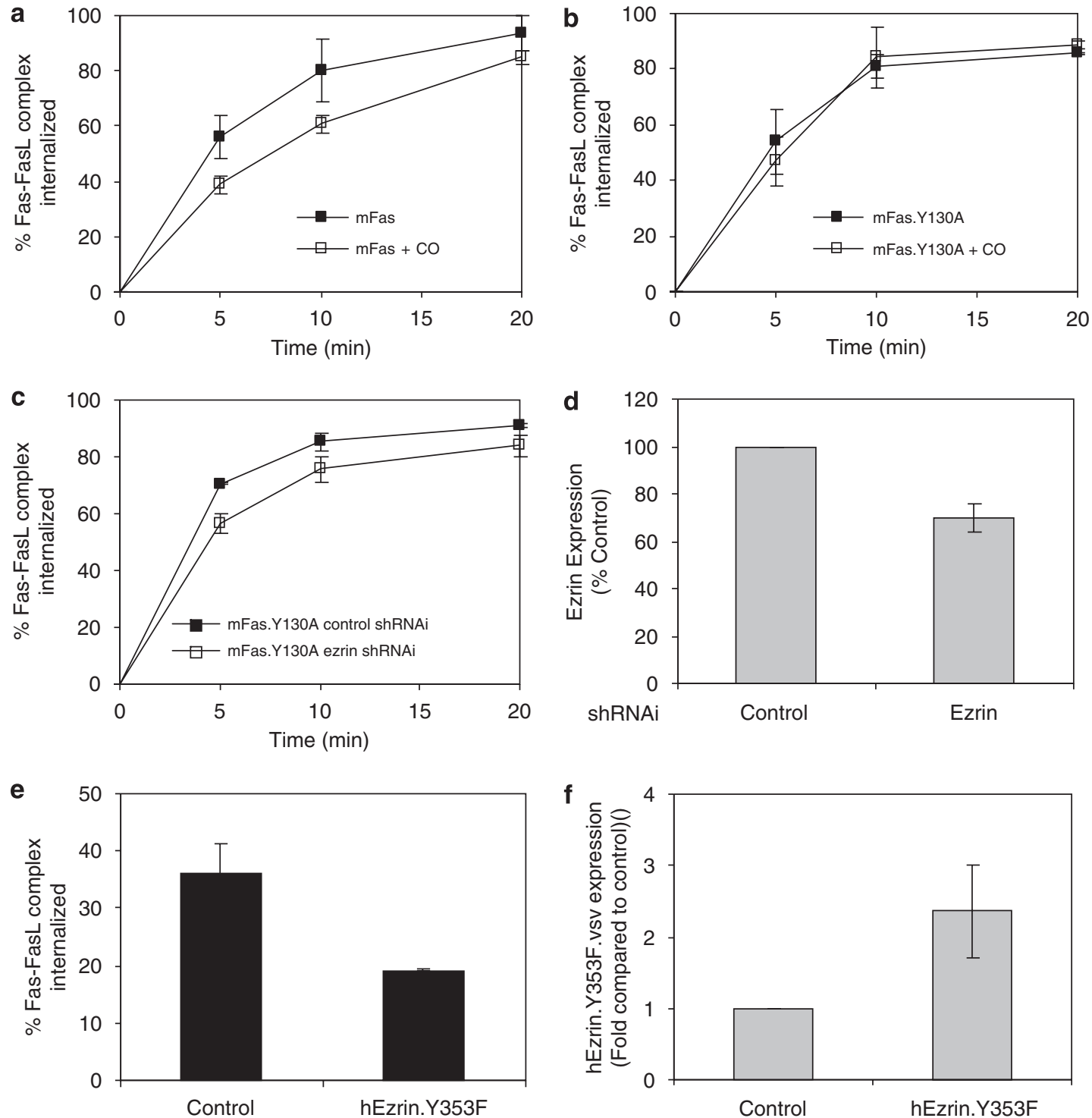

Figure 9 The internalization of GBM-defective Fas after activation is a chol-raft-independent but ezrin-dependent process. L1210.Fas (a) and L1210.mFas.Y130A (b) cells were untreated or treated with $4 \mathrm{U} / \mathrm{ml} \mathrm{CO}$ for $2 \mathrm{~h}$ before subjecting to Fas internalization assay (data shown as mean \pm S.D.). L1210.mFas.Y130A cells were stably transfected with indicated shRNAi before: (c) subjecting to Fas internalization, or (d) incubating with mouse anti-ezrin antibody in PBS containing $0.5 \%$ Tween 20 and $0.1 \%$ BSA, followed by FITC-conjugated anti-mouse Ig antibody and FACS analysis to assess the level of ezrin expression (shown by comparing the MFI of cells expressing ezrin shRNAi with those of the cells expressing control shRNAi). L1210.mFas.Y130A were stably transfected with pTER plasmid alone (control) or cotransfected with pTER and human ezrin.Y353F.VSVG plasmid (a kind gift from M Arpin; denoted as hEzrin.Y353F) before: (e) subjecting to Fas internalization assay ( $t=5$ min) or (f) incubating with mouse antiVSV glycoprotein antibody in PBS containing $0.5 \%$ Tween 20 and $0.1 \%$ BSA, followed by FITC-conjugated anti-mouse Ig antibody and FACS analysis to assess the level of VSV-tagged hEzrin. Y353F expression (shown by comparing the MFI of cells expressing hEzrin. Y353F with those of the control cells)

$\left.{ }^{3} \mathrm{H}\right]$-suramin $\left(10^{6}\right.$ counts/min per well), then washed five times with PBS. The radioactivity was determined using scintillation counter.

Cell death assay. Cell death triggered by crosslinked-FasL was done by incubating cells with Flag-tagged recombinant human FasL (rhFasL; Alexis) plus $1 \mu \mathrm{g} / \mathrm{ml}$ anti-Flag $\mathrm{Ab}(\mathrm{M} 2)$, at $37^{\circ} \mathrm{C}, 5 \% \mathrm{CO}_{2}$. Cells were then fixed with $70 \%$ ethano $\left(-20^{\circ} \mathrm{C}\right)$, washed in $38 \mathrm{mM}$ sodium citrate $(\mathrm{pH} 7.4)$, stained with propidium iodide with RNase A, and analyzed with a flow cytometer (FACSCalibur; Becton Dickinson). The proportion of apoptotic cells represented by the subG1 peak was determined and represented as percent of cell death. ${ }^{6}$ Cell death triggered by membrane-bound FasL was done by coculturing the target cells, for example, L12.10.mFas, with FasL-expressing cells, J16/Rapo-FasL (killer), or the FasL negative cells, J16/Rapo (control). ${ }^{6}$ Killer and control cells were prestained with
$5 \mu \mathrm{M}$ diacetate succinimidyl ester (CFSE) before the coculture to distinguish them from target cells during FACS analysis.

Boyden chamber motility assay. Cell culture inserts with polyethylene terephthalate (PET) membrane (8- $\mu \mathrm{m}$ pore size; BD Bioscience) were placed in 24-well plates containing $5 \%$ FBS-supplemented DMEM, without or with M2crosslinked FasL. The cells in serum-free DMEM without or with M2-crosslinked FasL $\left(6 \times 10^{5}\right.$ cells $\left./ 300 \mu l\right)$, were layered over the membrane insert (upper chamber). After 6 -h incubation, the insert was shaken for $30 \mathrm{~s}$ and the medium in the lower chamber was collected. The underside of the insert was then rinsed with PBS. The rinsing buffer was collected and pooled with the previously collected lowerchamber medium. The number of cells migrated from the upper chamber through the membrane was then determined with hemacytometer. 
Bromodeoxyuridine (BrdU) proliferation assay. Cells $\left(10^{6} \mathrm{cell} / \mathrm{ml}\right)$ were incubated for an indicated time in DMEM supplemented with $5 \%$ FBS without or with M2-crosslinked FasL and then pulsed with $10 \mu \mathrm{M}$ BrdU for $30 \mathrm{~min}$, washed with PBS, and fixed with $70 \%$ ethanol $\left(-20^{\circ} \mathrm{C}\right)$. Following DNA denaturation with $4 \mathrm{~N} \mathrm{HCl}$ and neutralization with $0.1 \mathrm{M}$ borax, cells were washed and incubated for 30 min with anti-BrdU in PBS with $0.5 \%$ Tween 20 and $0.1 \%$ BSA, followed by FITCconjugated anti-mouse $\mathrm{Ig}$. Amount of cells incorporating BrdU was then determined by FACS analysis.

DRM preparation. Post-nuclear supernatant from $3 \times 10^{7}$ cells was solubilized in $1 \mathrm{ml}$ buffer A (25 mM HEPES, $150 \mathrm{mM} \mathrm{NaCl}, 1 \mathrm{mM}$ EGTA, $10 \mathrm{mM}$ $\mathrm{Na}_{4} \mathrm{P}_{2} \mathrm{O}_{4}, 10 \mathrm{mM} \mathrm{NaF}, 5 \mathrm{mM} \mathrm{Na}_{3} \mathrm{VO}_{4}$, protease inhibitors cocktail) containing $1 \%$ Brii 98 (Aldrich) for $1 \mathrm{~h}$ on ice followed by addition of $2 \mathrm{ml}$ of $2 \mathrm{M}$ sucrose in buffer $\mathrm{A}$ before being subjected to a step sucrose gradient ultracentrifugation essentially as described. ${ }^{6}$ One-ml fractions were harvested from the top, except for the bottom fraction (no. 9) which contained $3 \mathrm{ml}$. To an equal volume of each fraction, Laeml buffer was added and the fractions were heated at $95^{\circ} \mathrm{C}$. Equal volume of each fraction was subjected to SDS-PAGE and immunoblotting.

Fluorescence correlation spectroscopy. The setup and measurements were performed as described using an Axiovert $200 \mathrm{M}$ microscope equipped with a Zeiss C-Apochromat $\times 40$ NA 1.2, water immersion objective and with excitation from a $488 \mathrm{~nm}$ line of an $\mathrm{Ar}+$-ion laser. ${ }^{6,17}$ The sample was illuminated with an excitation power of $<4 \mu \mathrm{W}$ at the back aperture of the objective lens. Autocorrelations were processed by a hardware correlator (ALV-GmBH) and data were analyzed with IgorPro (Wavemetrics).

Immunoprecipitation. Cells, unstimulated or stimulated with M2-crosslinked FasL, were sonicated in buffer A containing 1\% Nonidet-P40 and 10\% glycerol (lysis buffer) at $4^{\circ} \mathrm{C}$ and centrifuged to remove cell debris. The supernatant was immunoprecipitated as described ${ }^{6}$ using biotinylated $\mathrm{JO} 2$ anti-mFas antibody coupled to streptavidin-conjugated agarose + protein $\mathrm{G}$ sepharose beads.

Fas internalization assay. Fas internalization was analyzed as described. ${ }^{6}$ Briefly, except for cholesterol oxidase (CO) experiments where serum-free medium was used, cells were incubated with $200 \mathrm{ng} / \mathrm{ml}$ rhFasL with $1 \mu \mathrm{g} / \mathrm{ml}$ M2 or with M2 only (background-binding control) in DMEM supplemented with $5 \%$ FBS and $10 \mathrm{mM}$ HEPES on ice for $45 \mathrm{~min}$. After washing to remove unbound FasL, cells were then warmed and kept at $37^{\circ} \mathrm{C}$ for the indicated time to allow synchronized stimulation, or kept on ice (control unstimulated, $t=0 \mathrm{~min}$ ). Cells were then washed and stained with FITC-conjugated anti-mouse Ig and FasL on cell surface was detected by flow cytometry. To calculate the degree of Fas-FasL complex internalization, the median fluorescence intensity (MFI) of the background control cells (incubated with M2 only, $\mathrm{MFI}_{\mathrm{M} 2}$ ) was subtracted from the MFI of the FasL-bound cells $\left(\mathrm{MFI}_{\text {FasL }}\right)$ to obtain for each time point the absolute fluorescence of FasL bound on cell surface $\left(\mathrm{MFI}_{\mathrm{abs}}=\mathrm{MFI}_{\mathrm{FasL}}-\mathrm{MFI}_{\mathrm{M} 2}\right.$, e.g. $\mathrm{MFI}_{\mathrm{abs}, t=5}$ for 5-min stimulation). The $\mathrm{MFI}_{\mathrm{abs}, t=0}$ of the control cells which were kept on ice represents the total FasL-bound complex before internalization was commenced. The percentage of Fas-FasL complex internalized was calculated as $\left(1-\left(\mathrm{MFI}_{\mathrm{abs}, t=x}\right) /\left(\mathrm{MFl}_{\mathrm{abs}, t=0)}\right) \times 100\right.$

Immunofluorescent colocalization studies. Cells in DMEM supplemented with $5 \%$ FBS and $10 \mathrm{mM}$ Hepes were allowed to adhere to polylysine-coated coverslips for $45 \mathrm{~min}$ in 24 -well plate at $37^{\circ} \mathrm{C}$. The excess cell suspension was removed from the wells and medium with or without $200 \mathrm{ng} / \mathrm{ml} \mathrm{FasL}$ crosslinked with $1 \mu \mathrm{g} / \mathrm{ml}$ mouse anti-Flag (M2) was added to the cells on the coverslips. The cells were incubated at $37^{\circ} \mathrm{C}$ for $2 \mathrm{~min}$ to allow FasL-triggered stimulation, then fixed with $4 \%$ paraformaldehyde for $20 \mathrm{~min}$ at room temperature and subsequently permeabilized with $0.1 \%$ saponin. $\mathrm{CHC}$ and CALM were detected with corresponding primary antibodies followed by Alexa fluor 555-conjugated antigoat IgG secondary antibody. Fas-FasL complex was detected through M2 using Alexa fluor 488-conjugated anti-mouse IgG. Images were obtained using a laserscanning microscope (Zeiss LSM 510 META, equipped with an inverted Axiovert 200 ) with a $63 \times 1.4$ numerical aperture (NA) objective lens. All experimental and control images of the immunofluorescence data were collected using identical imaging settings. The assessment of colocalization of FasL/Fas complex and $\mathrm{CHC}$ or CALM was performed using NIH ImageJ Just Another Colocalization plug-in $(\mathrm{JACOP})^{39}$ (available at http://rsb.info.nih.gov/ij/plugins/track/jacop.html). JACoP provides the Pearson R correlation coefficient (PC), Manders' overlap coefficient (MOC), Mander's M1 and M2 coefficients, Li's intensity correlation quotient (ICQ) and Costes' randomization coefficients for a pair of images in the red and green channels. Detailed descriptions of these parameters can be found elsewhere. ${ }^{39}$ Briefly, PC measures the pattern similarities between two images. PC ranges from +1 (complete positive colocalization) to -1 (negative colocalization). MOC has a value ranging from 0 (no colocalization) to 1 (all pixels colocalize). It is insensitive to differences in signal intensities between the two channels, photo-bleaching or amplifier settings. Manders' M1 is the ratio of the summed intensities of pixels from the green channel for which the intensity in the red channel is above 0 to the total intensity in the green channel. M2 is the reverse of M1. M1 and M2 are independent of the intensity of fluorescence. Costes' randomization provides the indication whether the observed colocalization is purely because of chance or is it statistically significant. This method creates random images by shuffling pixels in the green channel and then calculating a new PC for each randomized image in the green channel (the image in the red channel is kept unchanged). The PC of the original image in the green and red channel is then compared with the PCs of the randomized green images and the significance ( $P$-value) is calculated. This $P$-value (expressed as percentage) is inversely correlated to the probability that the PC of the original image is obtained by chance. The ICQ value is calculated based on Li's intensity correlation analysis, which is considered a stable method for colocalization analysis as it allows the discrimination of coincidental events in a heterogeneous situation. ICQ varies from -0.5 (exclusion) to 0.5 (complete colocalization).

Acknowledgements. We thank Monique Arpin for kindly providing the hEzrin.Y353F plasmids, Hai-Tao He and Pierre Golstein for advices and helpfu discussions. This work was supported by institutional funds from the Centre National de la Recherche Scientifique (CNRS), grants from the Lique nationale contre le cancer (LNCC), the Association pour la Recherche contre le Cancer (ARC), the Emerald Foundation, the Agence Nationale de la Recherche (ANR), and a postdoctoral fellowship from the Susan G Komen Breast Cancer Foundation to KC.

1. Kischkel FC, Hellbardt S, Behrmann I, Germer M, Pawlita M, Krammer PH et al Cytotoxicity-dependent APO-1 (Fas/CD95)-associated proteins form a death-inducing signaling complex (DISC) with the receptor. EMBO J 1995; 14: 5579-5588.

2. Peter ME, Budd RC, Desbarats J, Hedrick SM, Hueber AO, Newell MK et al. The CD95 receptor: apoptosis revisited. Cell 2007; 129: 447-450.

3. Miaczynska M, Zerial M. Mosaic organization of the endocytic pathway. Exp Cell Res 2002 272: 8-14

4. Mayor S, Pagano RE. Pathways of clathrin-independent endocytosis. Nat Rev Mol Cell Biol 2007; 8: 603-612

5. Lee KH, Feig C, Tchikov V, Schickel R, Hallas C, Schutze S et al. The role of receptor internalization in CD95 signaling. EMBO J 2006; 25: 1009-1023.

6. Chakrabandhu K, Herincs Z, Huault S, Dost B, Peng L, Conchonaud F et al. Palmitoylation is required for efficient Fas cell death signaling. EMBO J 2007; 26: 209-220.

7. Falguieres T, Romer W, Amessou M, Afonso C, Wolf C, Tabet JC et al. Functionally different pools of Shiga toxin receptor, globotriaosyl ceramide, in HeLa cells. FEBS J2006; 273: $5205-5218$

8. Sharma DK, Brown JC, Cheng Z, Holicky EL, Marks DL, Pagano RE. The glycosphingolipid, lactosylceramide, regulates beta1-integrin clustering and endocytosis Cancer Res 2005; 65: 8233-8241.

9. Mahfoud R, Garmy N, Maresca M, Yahi N, Puigserver A, Fantini J. Identification of a common sphingolipid-binding domain in Alzheimer, prion, and HIV-1 proteins. J Biol Chem 2002; 277: 11292-11296.

10. Starling GC, Bajorath J, Emswiler J, Ledbetter JA, Aruffo A, Kiener PA. Identification of amino acid residues important for ligand binding to Fas. J Exp Med 1997; 185: 1487-1492.

11. Schneider $P$, Bodmer JL, Holler N, Mattmann C, Scuderi $P$, Terskikh A et al Characterization of Fas (Apo-1, CD95)-Fas ligand interaction. J Biol Chem 1997; 272 18827-18833.

12. Ling $\mathrm{H}$, Boodhoo A, Hazes B, Cummings MD, Armstrong GD, Brunton JL et al. Structure of the shiga-like toxin I B-pentamer complexed with an analogue of its receptor Gb3. Biochemistry 1998; 37: 1777-1788.

13. Fantini J. How sphingolipids bind and shape proteins: molecular basis of lipid-protein interactions in lipid shells, rafts and related biomembrane domains. Cell Mol Life Sci 2003 60: 1027-1032.

14. Weis WI, Drickamer K. Structural basis of lectin-carbohydrate recognition. Annu Rev Biochem 1996; 65: 441-473.

15. Hammache D, Pieroni G, Yahi N, Delezay O, Koch N, Lafont H et al. Specific interaction of HIV-1 and HIV-2 surface envelope glycoproteins with monolayers of galactosylceramide and ganglioside GM3. J Biol Chem 1998; 273: 7967-7971.

16. Yahi N, Sabatier JM, Nickel P, Mabrouk K, Gonzalez-Scarano F, Fantini J. Suramin inhibits binding of the V3 region of HIV-1 envelope glycoprotein gp120 to galactosylceramide, the receptor for HIV-1 gp120 on human colon epithelial cells. J Biol Chem 1994; 269 . 24349-24353. 
17. Wawrezinieck L, Rigneault $H$, Marguet $D$, Lenne PF. Fluorescence correlation spectroscopy diffusion laws to probe the submicron cell membrane organization Biophys J 2005; 89: 4029-4042.

18. Lenne PF, Wawrezinieck L, Conchonaud F, Wurtz O, Boned A, Guo XJ et al. Dynamic molecular confinement in the plasma membrane by microdomains and the cytoskeleton meshwork. EMBO J 2006; 25: 3245-3256.

19. Cahuzac N, Baum W, Kirkin V, Conchonaud F, Wawrezinieck L, Marguet D et al. Fas ligand is localized to membrane rafts, where it displays increased cell death-inducing activity Blood 2006; 107: 2384-2391.

20. Kusumi A, Nakada C, Ritchie K, Murase K, Suzuki K, Murakoshi H et al. Paradigm shift of the plasma membrane concept from the two-dimensional continuum fluid to the partitioned fluid: high-speed single-molecule tracking of membrane molecules. Annu Rev Biophys Biomol Struct 2005; 34: 351-378.

21. Phonphok Y, Rosenthal KS. Stabilization of clathrin coated vesicles by amantadine tromantadine and other hydrophobic amines. FEBS Lett 1991; 281: 188-190.

22. Macia E, Ehrlich M, Massol R, Boucrot E, Brunner C, Kirchhausen T. Dynasore, a cell-permeable inhibitor of dynamin. Dev Cell 2006; 10: 839-850.

23. Glebov OO, Bright NA, Nichols BJ. Flotillin-1 defines a clathrin-independent endocytic pathway in mammalian cells. Nat Cell Biol 2006; 8: 46-54.

24. Parlato S, Giammarioli AM, Logozzi M, Lozupone F, Matarrese $\mathrm{P}$, Luciani $\mathrm{F}$ et al. CD95 (APO-1/Fas) linkage to the actin cytoskeleton through ezrin in human T lymphocytes: a novel regulatory mechanism of the CD95 apoptotic pathway. EMBO J2000; 19: 5123-5134.

25. Li Q, Nance MR, Kulikauskas R, Nyberg K, Fehon R, Karplus PA et al. Self-masking in an intact ERM-merlin protein: an active role for the central alpha-helical domain. $J$ Mol Biol 2007; 365: 1446-1459.

26. Gautreau A, Poullet $P$, Louvard D, Arpin M. Ezrin, a plasma membrane-microfilament linker, signals cell survival through the phosphatidylinositol 3-kinase/Akt pathway. Proc Natl Acad Sci USA 1999; 96: 7300-7305.

27. Damke H, Baba T, van der Bliek AM, Schmid SL. Clathrin-independent pinocytosis is induced in cells overexpressing a temperature-sensitive mutant of dynamin. $J$ Cell Biol 1995; 131: 69-80.

28. Xavier R, Brennan T, Li Q, McCormack C, Seed B. Membrane compartmentation is required for efficient T cell activation. Immunity 1998; 8: 723-732.
29. Guo B, Kato RM, Garcia-Lloret M, Wahl MI, Rawlings DJ. Engagement of the human pre-B cell receptor generates a lipid raft-dependent calcium-signaling complex. Immunity 2000; 13: 243-253

30. Stoddart A, Dykstra ML, Brown BK, Song W, Pierce SK, Brodsky FM. Lipid rafts unite signaling cascades with clathrin to regulate BCR internalization. Immunity 2002; 17: 451-462.

31. Puri C, Tosoni D, Comai R, Rabellino A, Segat D, Caneva F et al. Relationships between EGFR signaling-competent and endocytosis-competent membrane microdomains. $\mathrm{Mol}$ Biol Cell 2005; 16: 2704-2718.

32. Abrami L, Liu S, Cosson P, Leppla SH, van der Goot FG. Anthrax toxin triggers endocytosis of its receptor via a lipid raft-mediated clathrin-dependent process. J Cell Biol 2003; 160: 321-328.

33. Abrami L, Leppla SH, van der Goot FG. Receptor palmitoylation and ubiquitination regulate anthrax toxin endocytosis. J Cell Biol 2006; 172: 309-320. e-pub 2006 January 2009

34. Rajendran L, Masilamani M, Solomon S, Tikkanen R, Stuermer CA, Plattner H et al. Asymmetric localization of flotillins/reggies in preassembled platforms confers inherent polarity to hematopoietic cells. Proc Natl Acad Sci USA 2003; 100: 8241-8246. e-pub 2003 June 25.

35. Schwede T, Kopp J, Guex N, Peitsch MC. SWISS-MODEL: an automated protein homology-modeling server. Nucleic Acids Res 2003; 31: 3381-3385.

36. Banner DW, D'Arcy A, Janes W, Gentz R, Schoenfeld HJ, Broger C et al. Crystal structure of the soluble human $55 \mathrm{kd}$ TNF receptor-human TNF beta complex: implications for TNF receptor activation. Cell 1993; 73: 431-445.

37. Fantini J, Garmy N, Yahi N. Prediction of glycolipid-binding domains from the amino acid sequence of lipid raft-associated proteins: application to $\mathrm{HpaA}$, a protein involved in the adhesion of Helicobacter pylori to gastrointestinal cells. Biochemistry 2006; 45 : 10957-10962.

38. Levy M, Garmy N, Gazit E, Fantini J. The minimal amyloid-forming fragment of the islet amyloid polypeptide is a glycolipid-binding domain. FEBS $J$ 2006; 273: 5724-5735

39. Bolte S, Cordelieres FP. A guided tour into subcellular colocalization analysis in light microscopy. J Microsc 2006; 224 (Part 3): 213-232.

Supplementary Information accompanies the paper on Cell Death and Differentiation website (http://www.nature.com/cdd) 